\title{
Ultrasound, CT and MRI Appearances of a Rare Symptomatic Laryngeal Chondrometaplasia: A Case Report
}

\author{
Ryan Ka Lok Lee ${ }^{1, *}$; Edmond Yuen Hok Yuen ${ }^{1}$; Victor James Abdullah ${ }^{2}$; Yolanda Yim Ping Lee ${ }^{1}$; \\ Anil Tejbhan Ahuja ${ }^{1}$ \\ ${ }^{1}$ Department of Imaging and Interventional Radiology, Prince of Wales Hospital, Chinese University of Hong Kong, Hong Kong, Chin \\ ${ }^{2}$ Department of Ear, Nose and Throat, United Christian Hospital, Hong Kong, China \\ *Corresponding author: Ryan Ka Lok Lee, Department of Imaging and Interventional Radiology, Prince of Wales Hospital, Chinese University of Hong Kong, Hong Kong, China. Tel: \\ +852-26321247, E-mail: leekalok2909@yahoo.com.hk
}

Received: September 22, 2012; Accepted: February 25, 2013

Symptomatic laryngeal chondrometaplasia is rare. To the best of our knowledge, there are only few case reports on laryngeal chondrometaplasia. The imaging appearance of this uncommon disease is even more rarely described. There are only two case reports describing its appearances in computed tomography (CT) and magnetic resonance imaging(MRI). Ultrasound(US) features have not been reported so far. This case report is to show the US, CT and MRI features of this disease entity to stress the role of imaging in this disease.

Keywords: Chondromatosis; MRI; Ultrasonography

\section{Introduction}

Symptomatic laryngeal chondrometaplasia is a rare entity. Metaplasia is the transformation of one differentiated tissue into another as a response to injury, but the exact etiology of the laryngeal chondrometaplasia is still unknown. The symptomatic laryngeal lesion is usually first discovered by fiber-optic laryngoscopy and subsequently diagnosed by biopsy. Cross-sectional imaging has been increasingly used to assess the exact origin and extent of the lesion. This case report is to describe the imaging appearances of this disease in ultrasound (US), computed tomography (CT) and magnetic resonance imaging (MRI) and to stress the role of imaging in the diagnosis of this disease.

\section{Case Presentation}

A 41-year-old female teacher presented with a 1-year intermittent hoarseness. She was a non-smoker and social drinker, and previously enjoyed good past health. The patient had no previous significant trauma to the throat or recent infection. There was no dysphagia, dyspnea, reflux symptoms or hemoptysis. Fiber-optic laryngoscopy revealed a small pedunculated erythematous nodule on the right false vocal cord that partially obscured the right true vocal cord. The left true and false vocal cords were normal and both vocal cords moved normally. Biopsy of this nodule showed chondrometaplasia. MRI was first performed to assess the origin and extent of the lesion and it revealed a T1-weighted hypointense and T2- weighted hyperintense contrast enhancing lesion within the lamina of the right thyroid cartilage (Figure $1 \mathrm{~A}$, $B)$. The lesion extended through the paraglottic fat space and protruded into the laryngeal lumen at the level of right false lumen (Figure $1 \mathrm{C}-\mathrm{E}$ ). This protruding luminal part of the lesion corresponded to the endoscopicallyidentified and biopsy-proven chondrometaplasia. There was no suspicious regional or cervical lymph node. Supplementary ultrasound and computed tomography were performed. The lesion was well-circumscribed and hypodense in CT without chondroid type calcification or any ossification (Figure 2). Ultrasound revealed the lesion as hypoechoic with marked internal vascularity (Figure 3). The patient was planned for surgical excision of the entire lesion.

\section{Discussion}

Laryngeal chondrometaplasia is not uncommon in postmortem study and is found in 1 - $2 \%$ of examined larynges (1). However, it is usually small in size (usually less than $1 \mathrm{~cm}$ in diameter) and thus asymptomatic (1). Symptomatic laryngeal chondrometaplasia is rare and seldom reported $(2,3)$. It may be difficult to differentiate laryngeal chondrometaplasia from the chondroid tumor such as chondroma or low-grade chondrosacroma either clinically, histologically or radiologically (2). The treatment for different chondroid tumors and chondrometaplasia is not the same. The laryngeal chondrometaplasia can

Copyright (C) 2015, Tehran University of Medical Sciences and Iranian Society of Radiology. This is an open-access article distributed under the terms of the Creative Commons Attribution-NonCommercial 4.0 International License (http://creativecommons.org/licenses/by-nc/4.0/) which permits copy and redistribute the material just in noncommercial usages, provided the original work is properly cited. 

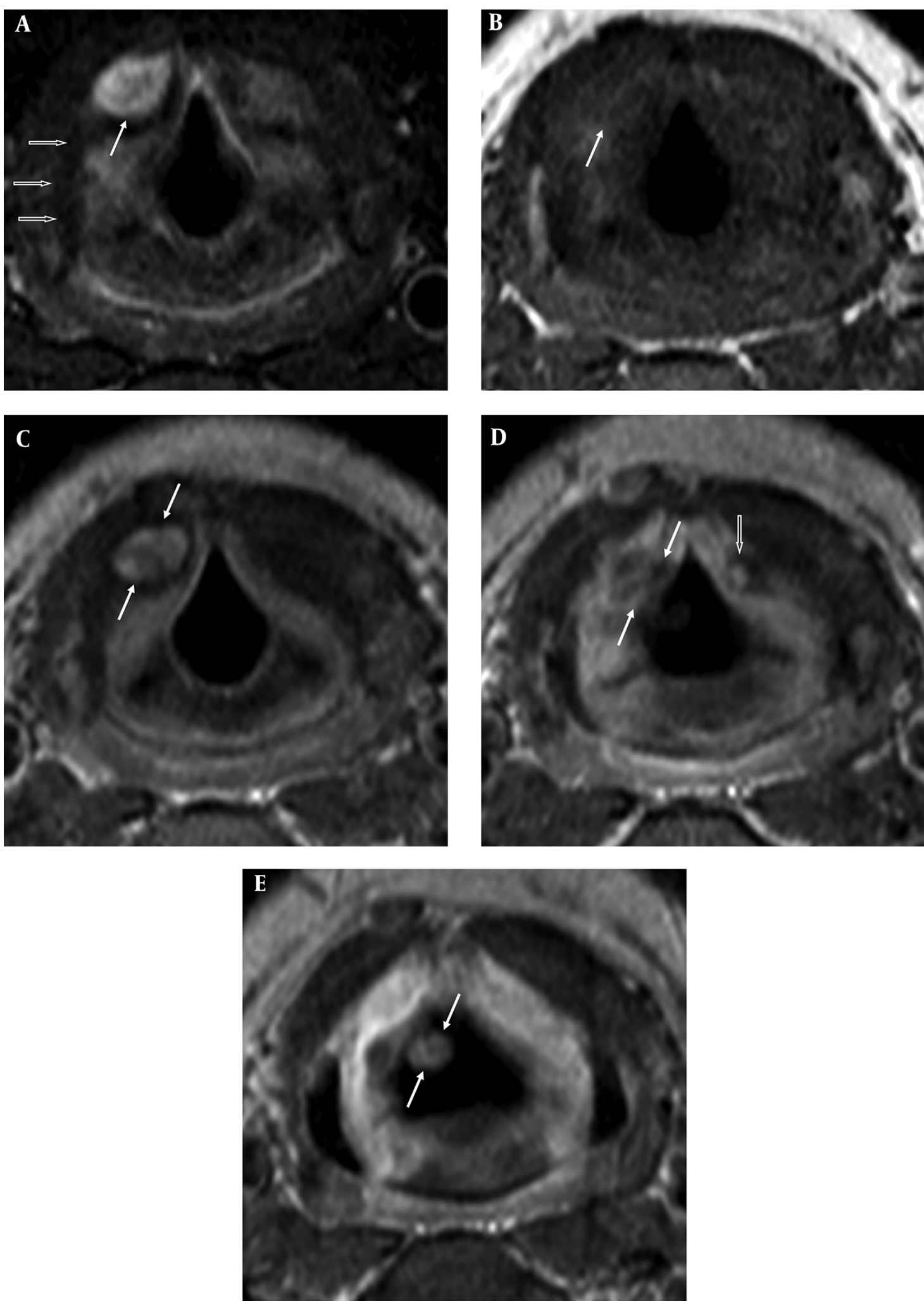

Figure 1. A 41-year-old woman who had intermittent hoarseness for one year. A, Axial T2-weighted MRI of the larynx with fat suppression technique reveals a T2-weighted hyperintense lesion (white arrow) within the lamina of the right thyroid cartilage (white open arrows). B, Axial T1-weighted MRI of the larynx shows that the lesion is T1-weighted hypointense (white arrow). Consecutive three slices of axial T1-weighted MRI of the larynx after gadolinium injection shows that the lesion (white arrows) extends from the thyroid cartilage (C), passes through the paraglottic fat space (D) and protrudes into the laryngeal lumen at the level of the false cord (E). A similar but smaller lesion is present within the lamina of the left thyroid cartilage. 
Lee RKL et al.

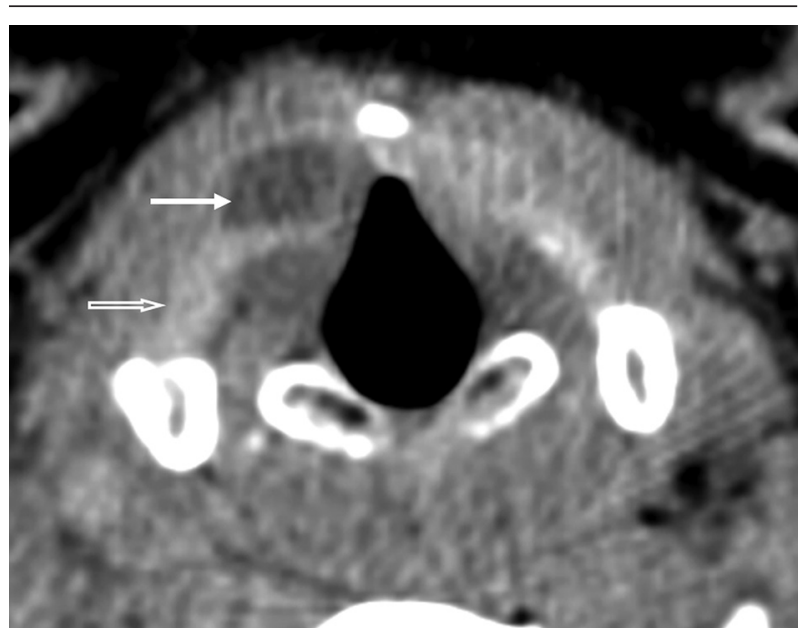

Figure 2. The corresponding axial non-contrast CT scan shows that the lesion is well-defined and hypodense. It is within the lamina of the right thyroid cartilage (white arrow). No chondroid matrix or calcification/ossification is present. There is a mild mass effect associated with displacement of the adjacent neck muscle and laryngeal mucosa but no invasion is visible. The open arrow shows the normal thyroid cartilage.

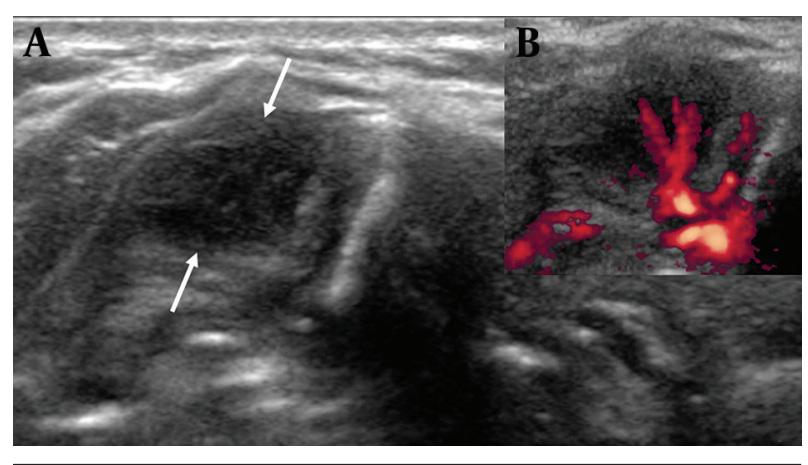

Figure 3. Axial grey-scale sonography (A) reveals homogeneous hypoechoic lesion within the right thyroid cartilage. No cystic change or calcification is identified. Power Doppler sonography (B) shows marked internal vascularity of the lesion.

originate from mucosa, submucosa, adjacent connective tissue, or cartilage of the larynx. It shows predilection for the posterior and mid-portions of the glottis, and for the ventricular bands (2). In the previous autopsy study, about $40 \%$ of them showed bilateral involvement (4). The clinical presentation of laryngeal chondrometaplasia is similar to the other laryngeal tumors. The symptoms include hoarseness, dyspnea, dysphagia or vocal fold palsy. It is postulated that previous laryngeal trauma or laryngo-tracheitis predisposes to the development of metaplasia in the larynx $(2,3)$. It is also suggested that the presence of these predisposing factors with progressive onset of symptoms may help in recognizing the chondrometaplastic nature of the lesion and distinguishing it from true laryngeal cartilaginous tumors which usually have no triggering factors $(2,3)$. Our patient is a school teacher with chronic repeated use of her voice. It is not clear whether the chronic overuse of the larynx may result in continuous irritation of the normal soft tissue of the larynx and thus the development of metaplasia. Microscopically, chondrometaplasia shows fibroblastlike cells surrounding the cartilage with progressive transition of these cells into central chondrocytes and presence of dense aggregation of elastic fibres in the stroma (3). The cartilage tissue resembles fibroelastic cartilage instead of hyaline cartilage that makes up most of the normal laryngeal cartilaginous structure except epiglottis (3). The above distinctive features differentiate chondrometaplasia from chondroma and chondrosacroma. Chondroma is relatively hypocellular and composed of uniformly spaced chondrocytes that lack unclear atypia while chondrosarcoma is characterized by a lobulated chondrocyte growth pattern, with invasive growth of neoplastic lobules into adjacent soft tissue (5). To the best of our knowledge, so far there are only two case reports describing the CT appearance of laryngeal chondrometaplasia $(2,6)$. In the first case report, the case described was similar to ours that the lesion originated from the lamina of the thyroid cartilage and bulged into the laryngeal lumen. The contrast CT revealed a rounded, well-circumscribed enhancing lesion. However, the precontrast CT was not shown or described in that report. In our case, the non-contrast CT showed no chondroid calcification which is typically present in chondroma and chondrosacroma (7). Although both chondroid tumor and chondrometaplasia can be well-defined and hypodense, chondroma and chondrosacroma may demonstrate invasion to the adjacent structure and may be differentiated from chondrometaplasia which usually results in only displacement rather than invasion to adjacent structures (7). However, the above features are not absolute in differentiating between chondrometaplasia and chondroid tumors (7). Biopsy is thus essential. In the second case report, the CT showed an ill-defined hypodense thickening and enhancement of the supraglottic soft tissue, predominantly involving the submucosa, along the greater cornu of the left side of the thyroid cartilage (6). No chondroid-type lesion or intraluminal extension was present. This was difficult to distinguish from other laryngeal tumors by the imaging alone in that case. In our case, US and MRI revealed no specific or characteristic features of chondrometaplasia to differentiate from other laryngeal tumors. Similar to the only available case report of chondrometaplasia with MRI findings, the lesion here is T1-weighted hypointense and T2-weighted hyperintense which are non-specific and indistinguishable from other laryngeal tumors (6). Cross-sectional imaging has been increasingly used in the assessment of the full extent of laryngeal chondrometaplasia that is usually first discovered in the fiber-optic laryngoscopy in symptomatic cases. As shown in our case, the small nodule seen in endoscopy only represents a part of the lesion that actually originates from a larger part within the thyroid cartilage. In comparison, MRI has better soft tissue resolution than CT 
and it is the imaging of choice in the assessment of the extent of chondrometaplasia.

US, CT and MRI features of this rare symptomatic chondrometaplasia have been described in this case report. Cross-sectional imaging, such as CT or MRI is useful in the assessment of the origin and extent of laryngeal chondrometaplasia and the plan for surgical approach. There are some reported CT features in differentiating chondrometaplasia from chondroid tumors, but these features are not specific or characteristic enough for the definite diagnosis. Biopsy is still essential for the final diagnosis and it excludes other laryngeal tumors.

\section{References}

1. Iyer PV, Rajagopalan PV. Cartilaginous metaplasia of soft tissues in the larynx. Case report and literature review. Arch Otolaryngol. 1981;107(9):573-5.

2. Orlandi A, Fratoni S, Hermann I, Spagnoli LG. Symptomatic laryngeal nodular chondrometaplasia: a clinicopathological study. $J$ Clin Pathol. 2003;56(12):976-7.

3. Hill MJ, Taylor CL, Scott GB. Chondromatous metaplasia in the human larynx. Histopathology. 1980;4(2):205-14.

4. Hsaio E, Thomson N, Allen J. Cartilaginous metaplasia of false vocal cord : case report and discussion. J Otolaryngol Soc Aust. 2003;6(1):27-9.

5. Lewis JE, Olsen KD, Inwards CY. Cartilaginous tumors of the larynx: clinicopathologic review of 47 cases. Ann Otol Rhinol Laryngol.1997;106(2):94-100.

6. Chaturvedi A, Kane SV. Laryngeal chondrometaplasia: a great mimic of chondrosarcoma. Indian J Pathol Microbiol. 2007;50(2):391-4.

7. Wang SJ, Borges A, Lufkin RB, Sercarz JA, Wang MB. Chondroid tumors of the larynx: computed tomography findings. Am J Otolaryngol.1999;20(6):379-82. 